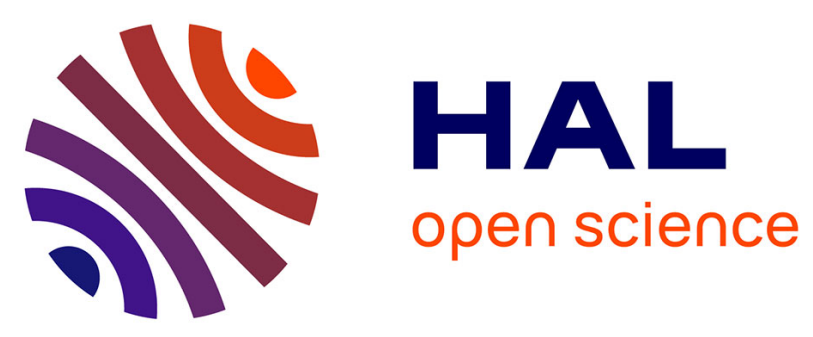

\title{
Un dispositif multi-groupe original en France. Articulation d'un grand groupe de partage d'expériences et de petits groupes thérapeutiques. Fonction thérapeutique et de formation
}

Marie-Frederique Bacque, Gilles Bertschy, Chloé Nicot, Renaud Beraldin, Anne Thevenot, Claire Metz

\section{To cite this version:}

Marie-Frederique Bacque, Gilles Bertschy, Chloé Nicot, Renaud Beraldin, Anne Thevenot, et al.. Un dispositif multi-groupe original en France. Articulation d'un grand groupe de partage d'expériences et de petits groupes thérapeutiques. Fonction thérapeutique et de formation. Revue de psychothérapie Psychanalytique de Groupes, 2019, n 72 (1), pp.163-175. 10.3917/rppg.072.0163 . hal-02991307

\section{HAL Id: hal-02991307 \\ https://hal.science/hal-02991307}

Submitted on 5 Nov 2020

HAL is a multi-disciplinary open access archive for the deposit and dissemination of scientific research documents, whether they are published or not. The documents may come from teaching and research institutions in France or abroad, or from public or private research centers.
L'archive ouverte pluridisciplinaire HAL, est destinée au dépôt et à la diffusion de documents scientifiques de niveau recherche, publiés ou non, émanant des établissements d'enseignement et de recherche français ou étrangers, des laboratoires publics ou privés. 
UN DISPOSITIF MULTI-GROUPE ORIGINAL EN FRANCE.

ARTICULATION D'UN GRAND GROUPE DE PARTAGE

D'EXPÉRIENCES ET DE PETITS GROUPES THÉRAPEUTIQUES. FONCTION THÉRAPEUTIQUE ET DE FORMATION

Marie-Frédérique Bacqué, Gilles Bertschy, Chloé Nicot, Renaud Beraldin, Anne Thévenot, Claire Metz

Depuis plus de six années, une équipe de psychologues et de psychiatres a mis au point, avec l'unafam (Union nationale des amis et familles de personnes malades psychiques), un dispositif original de groupes de parole. Il consiste en un soutien psychologique groupal de personnes souffrant de troubles de l'humeur et de leurs proches. À un grand groupe ouvert mensuel annoncé dans la presse locale et par voie d'affiche dans le service de psychiatrie de l'hôpital universitaire régional, sont proposés, en complément, trois petits groupes thérapeutiques fermés réservés respectivement aux personnes bipolaires et aux parents de jeunes adultes bipolaires (le groupe réservé aux conjoints de personnes bipolaires ne sera pas abordé ici).

L'articulation entre ces groupes permet à une personne, malade ou proche de malade, d'assister librement au grand groupe quand elle le désire et aussi longtemps qu'elle le souhaite. Elle peut alors passer un contrat thérapeutique oral avec un des petits groupes pour une durée

Marie-Frédérique Bacqué, professeure des universités, directrice de l'ea 3071, Faculté de psychologie, Strasbourg ; mfbacque@club-internet.fr

Claire Metz, maître de conférences hdr des universités, Faculté de psychologie, Strasbourg ; metz.claire@gmail.com

Anne Thevenot, professeure des universités, Faculté de psychologie, Strasbourg ; anne.thevenot@ unistra.fr

Renaud Beraldin, psychologue ; renaud.beraldin@club-internet.fr

Gilles Bertschy, praticien hospitalier chef de service, professeur des universités ; Service de Psychiatrie 2, Hôpitaux universitaires de Strasbourg, 1, place de l'Hôpital, 67091, Strasbourg ; Fédération de Médecine Translationnelle, Faculté de Médecine, Université de Strasbourg ; inserm u1114, Strasbourg ; gilles.bertschy@chru-strasbourg.fr

Chloé Nicot, ancien chef de clinique de l'université, assistant des hôpitaux ; Service de Psychiatrie 2, Hôpitaux universitaires de Strasbourg, 1, place de l'Hôpital, 67091, Strasbourg ; Fédération de Médecine Translationnelle, Faculté de Médecine, Université de Strasbourg; chloenicot@hotmail.com 
d'une année (dix séances) renouvelable. L'évaluation qualitative du dispositif a permis de valider son acceptabilité (évaluation subjective favorable), la richesse des thèmes traités et son intérêt dans le long terme (certains patients et proches continuent à participer aux groupes depuis leur ouverture).

Dans cet article, nous développons les différentes fonctions de ces groupes, la place des interlocuteurs et participants, les techniques d'animation référées à la psychanalyse de groupe (Anzieu, 1982), l'intérêt de leur apport thérapeutique et social. Nous exposons également l'enseignement informel constitué par le grand groupe qui, outre les personnes bipolaires et leurs proches, reçoit aussi des étudiants en psychologie, des internes en psychiatrie, des psychiatres libéraux, des médecins généralistes et des infirmiers. Les groupes ne forment donc pas seulement un lieu de restauration d'un lien social pour des personnes souvent en marge de la société mais aussi une sorte de "mixité sociale » entre personnes touchées par la maladie bipolaire et les soignants. La «bonne distance » des animateurs leur permet d'exercer une médiation du discours et de l'expérience ainsi que l'interprétation des paroles ou comportements.

Un dernier mot sur le terme « bipolaire », adopté aujourd'hui par la plupart des patients. Il est souvent discuté en groupe comme l'histoire de la maladie maniaco-dépressive. Le désir pour certains de trouver une identité est si grand que la plupart énoncent « Je suis bipolaire », ou encore " Chez les bipolaires... Nous, les bipolaires... », etc. Nous avons choisi de parler de " personne bipolaire », bien que nous utilisions encore parfois l'expression " malade bipolaire ». Nous sommes attentifs aux étiquettes qui figeraient les patients et leurs proches. Leur ambivalence est cependant perceptible : nombreux se plaignent de « n'avoir un diagnostic qu'en moyenne au bout de dix ans », mais la plupart rejettent la stigmatisation dont ils font l'objet. Cette contradiction constitue un objet habituel de discussion dans les groupes, quels qu'ils soient.

1a maladie bipolaire au quotidien

D'après l'eufami (European Federation of Associations of Families of People with Mental Illness), il y aurait 25 millions de familles de malades psychiatriques en Europe. Ces familles sont principalement composées de conjoints et de parents de personnes présentant un " handicap psycho-social » conséquent comme dans les schizophrénies et les troubles bipolaires. Les patients, souvent fortement dépendants de leurs parents, le restent parfois jusqu'à la fin de leurs jours. P. Federn écrivait dès les années 1950 que «l'alliance avec la famille est un facteur plus déterminant dans l'évolution, que les caractères intrinsèques de la pathologie ». Les groupes de parents en témoignent. 
Les parents de malades psychiatriques ont, quant à eux, bien changé (Metz et coll., 2017). Âgés de 40 à 60 ans et plus, ils appartiennent à des générations qui bénéficient des informations mondialisées par Internet. Parmi ces parents, certains sont prêts à se mobiliser pour ne plus être isolés et exprimer leur point de vue sur les réseaux sociaux ou dans des groupes (Mickelson, 1997). Ainsi, lors d'une session du grand groupe de 2016, les parents d'une jeune femme ont relaté comment ils avaient parcouru $10000 \mathrm{~km}$ pour rechercher leur fille bipolaire, partie brusquement avec un homme rencontré lors d'une soirée. Ils n'ont pas pu la ramener mais ont constaté qu'elle était devenue quasiment sdf et très isolée. Ils sont venus confier au grand groupe leurs difficultés et leurs interrogations : "Même si elle est majeure, peut-on abandonner tout contact avec elle ? Est-ce vraiment ce qu'elle désire ? La maladie la rend vulnérable. Nous souhaitons encore la protéger. »

Politiquement, le lobbying et les class-actions, la remise en cause $\mathrm{du}$ paternalisme médical et l'avènement de l'empowerment du patient dans de nombreux champs de la maladie chronique, donnent un statut différent à la famille qui, après une forme de passivité fataliste, exprime une volonté d'action et de reconnaissance (Bacqué, 2012). La proximologie, « science du proche », propose de faire de l'aidant familial, un « partenaire du soin » (Joublin, 2005). Adoptée en oncologie, elle a installé en psychiatrie, un véritable « trialogue », comme en Autriche ou en Allemagne ${ }^{1}$, où le soin passe de plus en plus par le dialogue en triade patient-proche-médecin/soignant (Jarchov-Jadi et coll., 2013 ; Wallcraft et coll., 2011).

Sur le plan sociologique, l'institutionnalisation de la personne souffrant de troubles psychiatriques est fréquemment liée à un épisode aigu mais, lors des périodes intercritiques, le patient " chronicisé », de retour chez lui, se trouve confronté à des difficultés pour suivre les soins. Le constat d'une certaine précarisation des malades qui ne respectent, par défaut de motivation ou de compréhension, ni leurs rendezvous ni les posologies des traitements, est commun aux pays développés. Livrés à eux-mêmes, certains patients français délaissent d'autant plus facilement leur accompagnement thérapeutique que la sectorisation psychiatrique dépend d'une cartographie complexe (Bauer et coll., 2011). De plus, les soins médicaux sont rarement doublés d'une psychothérapie qui permettrait, a minima, d'associer un soutien psychique face aux différents effets physiologiques et psychosociaux, de la maladie chronique. Enfin, l'intérêt sociétal pour la maladie mentale et les troubles psychiques est paradoxalement trop faible ou seulement stigmatisé au travers d'affaires médico-légales qui ont un fort retentissement dans les médias (Henckes, 2015).

1. Le Weddinger Modell propose à Berlin l'autodétermination du patient en impliquant systématiquement les proches dans l'échange avec les soignants. Mais ce changement de paradigme conviendra-t-il à toutes les cultures et surtout à toutes les cultures psychiatriques? 
Sur le plan économique, l'hospitalisation n'est plus l'unique issue de la psychose chronique. Les médicaments permettent un contrôle plus distancié des patients dorénavant suivis en ville ou en consultation externe. Certains patients se transforment même en patients-experts ou en médiateurs de santé/pairs, comme à l'hôpital Esquirol à Paris ou à Marseille (Le Cardinal et coll., 2013) et deviennent alors des proches pour les malades. La France rejoint ici le mouvement américain du " rétablissement » (recovery) dans lequel le patient, ou usager, est reconnu comme le meilleur expert de sa maladie. Le " rétablissement » n'a cependant pu exister qu'à partir des travaux de l'école psychanalytique sud-américaine et particulièrement ceux d'E. Pichon-Rivière qui situait les patients au cœur du dispositif de soin (Jaitin, 2002). Si les grandes métropoles françaises proposent plus fréquemment l'autonomisation des malades, les régions sont encore en quête de systèmes plus adaptés à leur culture locale. Ainsi l'étude menée à Marseille (Zendidjian et coll., 2012) montre combien ces interactions malades/ proches ont d'effets limitatifs sur la vie (évaluée via la qualité de vie) de personnes qui, a priori, n'avaient ni symptômes ni difficultés psychiatriques (Nicot, 2015).

possibilitÉs de soutien familial dans les soins psychiatriques en france

Compte tenu de la désinstitutionnalisation des patients et conséquemment des durées d'hospitalisation écourtées, les familles sont davantage impliquées dans le soin tout en restant démunies par le manque d'information sur la pathologie. Divers types de soutien des familles existent cependant depuis 1960 grâce à l'unapei (Union nationale des associations de parents, de personnes handicapées mentales et de leurs amis). Cependant, ces « formations » apportent des connaissances mais pas d'élaboration psychique des changements.

Les processus de changement sont décrits dans différents types de thérapie familiale (Perlick et coll., 2010 ; M'Bailara et coll., 2014), suivant le modèle systémique développé dès les années 1970. Les petits groupes des thérapies multifamiliales font aussi penser au dispositif de Lugar de vida au Brésil, destiné aux familles d'enfants autistes (Machado-Kupfer, de Lajonquière, 2014). Aujourd'hui, des groupes de parole et de partage, des groupes de psychoéducation (Fristad et coll., 2003 ; M'Bailara et coll., 2014 ; Pavuluri et coll., 2004) empruntant au modèle cognitiviste centré sur la résolution de problèmes (GexFabry et coll., 2015 ; Pavaluri et coll., 2004 ; West et coll., 2009) sont principalement développés, alors que les groupes psychodynamiques ont depuis longtemps fait leur preuve (Brun et coll., 2016).

La thérapie multifamiliale initiée par S. Cook-Darzens (2007) dans le cadre de l'anorexie mentale porte de nombreuses promesses. Il s'agit de rassembler dans un contexte thérapeutique, plusieurs familles au lieu d'une seule. Le thérapeute devient ici un facilitateur, garant d'un 
système " supra-thérapeutique » dans lequel les familles deviennent co-thérapeutes entre elles (Marchand, 2008).

L'originalité de notre groupe multifamilial est liée au référentiel psychanalytique. D'une part, cette épistémologie indique la prise en compte éthique de la subjectivité des personnes en présence, patients, proches ou thérapeutes. D'autre part, la reconnaissance de l'inconscient freudien et des stades du développement apporte la compréhension des phénomènes à l'œuvre : régression, identification, déplacement, condensation, sont retrouvés couramment dans les séances de groupe et évoluent avec le temps.

prÉsentation du dispositif grand groupe de partage/petits groupes thÉrapeutiques

Notre présentation est qualitative mais nous disposons du compte rendu de chaque séance, véritable synthèse des différents thèmes abordés chronologiquement. La prise de notes est toujours réalisée par un psychologue observateur qui n'anime pas le groupe. La soixantaine de comptes rendus recueillie en six ans forme ainsi un véritable réservoir clinique objectif de ce qui est partagé dans chaque grand groupe.

\section{Historique et formation des animateurs}

Deux universitaires, un psychiatre clinicien spécialiste des patients souffrant de troubles bipolaires et une psychologue psychanalyste conduisant, depuis plus de vingt-cinq ans, différents types de groupes ${ }^{2}$, sont à l'origine du grand groupe. Ils considèrent qu'une relative opposition entre tenants de la psychanalyse et de la psychiatrie constitue un motif dépassé (Masson et coll., 2015) et conviennent d'unir leurs intérêts de façon créative pour les patients. Les groupes ainsi lancés respectent le « terrain », celui de l'histoire des institutions psychiatriques d'une région française et d'une coopération ancienne avec une association, l'unafam. Une collaboration originale de psychiatres et de psychologues/psychanalystes dans le cadre associatif de l'unafam voit donc le jour en proposant une alliance de travail systématique entre psychologues et psychiatres formant chaque dyade d'animateurs des groupes.

\section{Le grand groupe}

Selon Bleger (1967), « le groupe s’organise autour de dépôts syncrétiques ». Ces « dépôts », éléments psychiques les plus primitifs et

2. Adultes, enfants et adolescents, personnes en deuil, après un psychotraumatisme, ou encore professionnels en groupes Balint (soignants, médecins et psychologues en formation ou en exercice). 
les plus indifférenciés de l'institution familiale trouvent enfin un réceptacle dans le groupe thérapeutique. Ils ne sont pas seulement contenus et mis en résonance, mais sont reliés grâce à l'activité de pensée et de parole des animateurs.

Le grand groupe de partage " tout-venant» est une sorte de hubplatform qui permet à tous, patients et proches, de venir depuis 2010 une fois par mois échanger sur leurs expériences. Ce grand groupe est libre d'accès, sans obligation de revenir. Les animateurs rencontrent donc, à chaque séance, un groupe inconnu et imprévisible. Ce sont des parents qui viennent d'hospitaliser leur enfant; des « patients », qui sont " déprimés ou ont eu une crise maniaque " mais dont l'investigation diagnostique n'a pas encore abouti. L'équipe des animateurs ne s'appuie pas sur une nosographie pour permettre la participation mais sur l'expérience subjective et médicale de personnes « se disant bipolaires ». D'autres participants ont, au contraire, « l'assurance » d'être bipolaires et bénéficient d'un suivi psychiatrique depuis de longues années. Tous, quelle que soit leur préconception d'avoir besoin de venir au grand groupe, sont les bienvenus. Les grands groupes deviennent ainsi parfois de " très " grands groupes, atteignant jusqu'à cinquante personnes.

\section{Les petits groupes}

Destinés aux personnes confrontées à la maladie bipolaire, deux petits groupes thérapeutiques fonctionnent en parallèle depuis 2013 et s'adressent, l'un, exclusivement aux patients, et l'autre, exclusivement aux parents de patients. Un groupe thérapeutique de conjoints de personnes bipolaires est en cours d'évaluation.

\section{Formation des étudiants en psychologie, des internes en psychiatrie, de soignants libéraux et étrangers}

Comme E. Pichon Rivière, qui avait élargi le dispositif du " groupe de soin » à l'apprentissage, nos groupes n'ont pas qu'une fonction thérapeutique. Ils ont aussi une fonction de formation. Étudiants en psychologie et internes en psychiatrie y sont conviés, au moins une fois dans leur cursus, sans aucune obligation. Ces temps de visite formatrice font l'objet d'une reprise avec l'un des animateurs du groupe. La dureté de certains propos, l'expression émotionnelle souvent crue, certains comportements hypomanes ou sous l'influence de traitements médicamenteux, requièrent en effet une élaboration Enfin, le partage de l'animation entre psychiatre et psychologue est un signal fort vis-à-vis de " la faculté », en l'occurrence, celle de médecine pour les psychiatres du chru et celle de psychologie pour les psychologues. L'objectif ici est d'éviter le clivage entre une psychiatrie prescriptrice et une psychologie psychothérapeutique. 
Dans les groupes, les psychiatres écoutent et interprètent, tout comme les psychologues qui ont des connaissances diagnostiques et pharmacologiques. Tous partagent l'intérêt général pour le processus intersubjectif visible dans les groupes.

Certains psychiatres installés en libéral dans la région visitent aussi le grand groupe. D'autres, (Japon, Pérou) ont également fait un passage, à chaque fois explicité au grand groupe qui a toujours accepté avec intérêt ces observations. Une journaliste du Monde a écrit un article dans son journal après l'une de ces visites, suivie de l'interview de quelques participants (Laronche, 2011).

Cadre thÉorique et fonctionnement psychodynamique du grand groupe

Notre grand groupe est inspiré des « large groups » ou « community meetings » développés par Maxwell Jones, inspiré par Wilfred Bion et Siegmund H. Foulkes. Ces grands groupes originaires du RoyaumeUni réunissaient patients et équipes soignantes dans les années 1960, avec des méthodes d'animation inspirées de la psychanalyse mais aussi de la théorie des systèmes et de la sociothérapie. E. Pichon Rivière avait aussi suivi l'école anglaise (Meltzer, 1967) pour tirer sa théorie des trois $\mathrm{D}$ : dans tout processus de maladie mentale, il y a un dépositaire de la maladie, le patient, qui fonctionne comme un portevoix du groupe familial, qui, lui, est le déposant des fantasmes. Pour soigner le malade et diminuer la ségrégation, produit du clivage et de la dénégation, la famille essaie d'élaborer l'angoisse produite par la maladie (Jaitin, 1995). Le membre « le plus fort du groupe familial » est, selon Pichon Rivière, le patient, parce qu'il porte le poids du conflit en l'assumant inconsciemment. Ceci procure à l'ensemble du groupe une économie psychique. Le patient, "porte-voix » de la maladie, énonce le drame par son histoire personnelle et révèle l'implicite groupal. Notre grand groupe patients-proches rejoue les échanges famillemalade, même si ce ne sont pas les familles entières qui sont présentes, mais des malades et des proches.

Les « large groups » permettaient des échanges sur les pratiques soignantes et leur réception par les patients. Dans notre clinique, nous avons augmenté les catégories de participants. En effet, si les parents d'aujourd'hui ont un rôle qui dépasse largement leur lien de filiation, leur besoin de confronter leur expérience à celle des autres parents et des patients est très différent de celui des soignants. Les soignants sont formés et non liés affectivement à leurs patients. Les parents et les autres patients exercent plus directement le «mirroring 》 développé par Winnicott d'après le concept de Foulkes : reconnaître chez les autres membres du groupe, les parties projetées de soi en eux. Le travail de l'animateur (ou « conductor» selon Foulkes, 1975) consiste à pointer cet échange par projection/identification entre les différentes populations. Les règles de fonctionnement sont rappelées à chaque 
début de grand groupe : cadre, engagement, confidentialité, respect d'autrui et liberté de participation.

Quelques situations typiques illustrant six années d'exercice : parent qui monopolise la parole et, devant les interventions de l'animateur, n’hésite pas à dire qu'il est venu " très égoïstement chercher des solutions pour lui et sa famille ». Long échange multiple où tous les patients se transmettent la parole pour décrire par le menu leur tentative de suicide suivi par un silence mortifère que l'animateur brise pour permettre, après un temps de latence, l'élaboration de ces angoisses de mort aboutissant au passage à l'acte. Patient en accès maniaque, très agité, qui allume une cigarette dans le groupe. Patient très déprimé à qui l'un des animateurs propose, pendant la séance, de lui parler à la fin de celle-ci. Autre patient hypomane qui entre et sort à plusieurs reprises. Agression verbale d'une des patientes bipolaires sur sa tenue et sa manucure, jugée par une participante comme non représentative de la dépression qu'elle allègue. Tous ces passages à l'acte sont généralement repris dans le groupe par les animateurs mais peuvent effrayer les patients, parents et conjoints novices. Certains patients, venus avec leurs parents, téléphonent aux animateurs parce qu'ils ne s'identifient pas aux membres du groupe et demandent des adresses de psychiatres et de psychothérapeutes.

Cadre thÉorique et fonctionnement psychodynamique des petits groupes

Les petits groupes, ou " réunion d'un ensemble de personnes dans un même lieu, un même temps, afin d'accomplir une tâche commune dans un même but ", sont structurés en référence aux travaux de Foulkes, de Bion, d'Anzieu et de Neri. La dynamique de partage installe un transfert des patients sur le groupe et une mobilisation des défenses. Le rappel inattendu avec des expériences passées enfouies et la réalité de la maladie jusque-là évitée ou déniée, exige de la part des professionnels une intégration de leurs propres mouvements psychiques, ainsi que le repérage du fonctionnement psychique de chacun des participants. Les animateurs sont des facilitateurs de la discussion, mais ils sont aussi, par leur connaissance de la maladie bipolaire (psychologue/analyste et psychiatre), des référents qui reprennent les idées fausses qui, par le dialogue, peuvent être révisées. De même, si la détresse émotionnelle apparaît très souvent, l'objectif des animateurs n'est pas de la faire disparaître mais de permettre son élaboration et son intégration. La facilitation de l'autonomie est une valeur centrale des animateurs qui, par l'accueil bienveillant de tous, permet une alliance thérapeutique. L'échange basé sur les libres associations, la confrontation des points de vue, l'interprétation des éléments conscients et inconscients, enfin la perlaboration, sont possibles grâce aux dix séances annuelles et au bilan du travail effectué avant la séparation des vacances d'été. 
Les règles sont :

- le respect de l'autre par l'abstention de jugement de valeur à l'intérieur comme à l'extérieur du groupe ;

- la responsabilité par l'engagement à venir à toutes les séances (prévues pour une année) ;

- la réactivité, par l'écoute des autres et la participation en travaillant sur le matériel apporté par les membres, même si le désir est plutôt de libérer ses propres émotions ;

- la confidentialité par la non-divulgation à l'extérieur de ce qui se dit dans le groupe.

Depuis quatre années, la plupart des participants sont restés au moins un an, cinq sont présents depuis le début. Une toute jeune femme a débuté en septembre de l'année précédente et s'est parfaitement intégrée malgré la prise de conscience d'une maladie qui ne peut pas être guérie mais stabilisée avec des traitements. Deux patients ont choisi d'arrêter leur traitement avec l'accord de leur psychiatre et échangent avec le reste du groupe sur cette décision controversée. Le groupe forme une véritable enveloppe dans laquelle les patients parlent librement de ce qu'ils ressentent profondément. Ce lieu unique n'est pas qu'une structure de décharge des affects, au contraire, une véritable perlaboration dégage une autonomie de la pensée chez chaque membre qui en vient à assumer des choix de vie plus personnels. Le petit groupe est aussi un espace à partir duquel peuvent circuler d'autres propositions. Des activités extérieures (sans les animateurs) sont régulièrement organisées par certains membres qui souhaitent élargir le partage au-delà des affects.

Le petit groupe de parents montre aussi leur souhait de partager leurs vécus souvent empreints de sentiments de découragement, de désespoir et d'impuissance face à la maladie chronique de leur enfant bipolaire. Cinq parents sont présents depuis quatre ans. Certains viennent en couple, trois d'entre eux ont assisté aux séances durant la quatrième année. Les participants sont passés de neuf à quinze en 2017. Les thèmes spontanés sont : l'insertion professionnelle et les études continuées ou interrompues du jeune adulte, l'insertion sociale, l'insertion familiale (place du jeune dans la famille élargie), l'impact sur la fratrie, l'entrée dans la maladie et le souvenir traumatique de l'hospitalisation sous contrainte, l'étiologie de la maladie et ses représentations, la prise en charge et les traitements de la maladie, les soins, les affects des parents tels que : souffrance, usure, intranquillité, mécanismes défensifs face à la maladie et à l'instabilité de leur enfant, vécu subjectif des parents de la souffrance de leur enfant, sentiment de culpabilité, indépendance de l'enfant, son avenir (fonder une famille) et après la mort de ses parents, suicide ou tentative de suicide.

Le groupe des parents d'adultes « bipolaires » a une fonction d' " enveloppe psychique », de contenant des sentiments de détresse liés à la maladie chronique de leur enfant. Leur culpabilité, leur 
impuissance à l'aider, parfois leur exaspération, ou leur haine, trouvent ainsi une écoute contenante et symbolisante comme dans l'extrait suivant :

$C$ : Elle dit qu'elle est angoissée, déprimée, mais elle ne sait pas pourquoi. Quand elle était deux semaines chez nous, elle était imbuvable, c'était terrible. À l'hôpital, c'est aussi difficile, bon, là-bas, elle a plus de confort mais elle s'ennuie.

$G$ : Elle est encore dans le trou, ça va remonter.

$L$ : Ça fait du bien d'en parler.

ou lors de cet autre extrait :

$L$ : À la fête de Noël il y a une angoisse terrible.

$F:$ Ah, moi aussi.

$C$ : Moi aussi.

Lu : J'en ai marre de leur expliquer à la famille.

Li : Ça parle de carence.

$\mathrm{Lu}:$ De nunuche.

Li : Il y a le passif qui ressort dans les familles, entre la poire et le fromage.

Lu : Les neveux et nièces ont peur que leurs enfants soient pareils.

$C$ : Pareil. Ils se demandent ça vient de qui (la bipolarité).

$G$ : Elles m'accusent de l'avoir chouchoutée.

Lu : Nous aussi. On étouffe, on ne sait pas quoi dire...

Comme dans le groupe de patients, l'espace psychique des parents ne se limite pas à l'expression des affects, il revisite les représentations de la maladie, la reconnaissance par leurs pairs de leurs « trouvailles » pour faire face aux variations de l'état psychique de leur enfant. Soutenus par le groupe, accompagnés par les animateurs, les parents peuvent exprimer avec cynisme parfois, agressivité ou humour, leur ambivalence pour leur enfant dont le degré de dépendance, psychique et/ou matérielle, reste important.

Le grand groupe permet aux parents, éventuellement accompagnés de leur enfant adulte, de dédramatiser les moments de crise en écoutant les témoignages des autres familles. L'articulation grand groupe/petit groupe de parents permet à ces derniers de trouver une écoute et de réélaborer ces expériences traumatisantes, ainsi :

$G$ : Vous n'allez jamais dans le grand groupe?

$C: S i$, depuis six mois, ils sont intéressants les témoignages des gosses, c'est un échange d'expériences. L'un était très exalté. Vous êtes venue?

G : Oui, ma fille en 2000... Elle a fait une dépression pendant un an puis elle a remonté, elle est venue dans le grand groupe, elle a vu qu'il y avait beaucoup de jeunes. C'était un soulagement. Elle prend du recul, mais c'est un long travail d'acceptation.

L : Oui. 
$G$ : Elle ne voit pas d'avenir, elle dit «je me marierai pas, je veux pas de gamin dans cette galère », à 30 ans.

Conclusion

Notre dispositif de soin multi-groupe propose, de façon originale, un partage des expériences des différents acteurs de la maladie bipolaire. Le caractère chronique de cette pathologie la rend en effet aussi difficile à supporter pour les parents que pour leur enfant. Ces derniers se retrouvent donc ensemble dans un grand groupe qui forme une nouvelle déclinaison des "large groups » développés par Maxwell Jones, dans la lignée de Wilfred Bion et Siegmund H. Foulkes. Deux types de groupes thérapeutiques ont été créés pour permettre un travail psychique plus approfondi avec les personnes bipolaires et les parents de personnes bipolaires. Les parents d'adultes bipolaires, souvent exclus du dispositif de soin, participent avec persévérance et fidélité aux petits groupes organisés à leur demande. Le petit groupe multifamilial de parents semble répondre à leur besoin crucial. Il installe un soutien émotionnel qui remobilise les ressources psychiques et limite le débordement par les angoisses et la lassitude. Il permet à ces parents de continuer à aider les patients eux-mêmes. Si parents et patients sont dans des groupes thérapeutiques fermés, le grand groupe reste ouvert et reçoit toutes ces personnes quand elles le souhaitent et sans restriction. Le grand groupe devient alors un lieu de partage social de l'expérience " à la Pichon Rivière », tout en accueillant les questionnements et les aléas de la maladie bipolaire. Les différents groupes de partage émotionnel multi-patients et multifamilial d'inspiration psychanalytique s'inscrivent dans un dispositif multi-groupe, multi-focal et plurithéorique dont nous poursuivons le dépouillement des données. Ce dispositif constitue une innovation dans le paysage évolutif des thérapies psycho-sociales pour les personnes bipolaires et leur entourage.

bibliographie

anzieu, d. 1982. Le travail psychanalytique dans les groupes, Paris, Dunod.

bacqué, M.-F. 2012. "Troubles de l'humeur : rôles et souffrances des aidants », Communication au colloque «Psychoses à travers les âges », Strasbourg.

bauer, r. ; gottfridsen, g.u. ; binder, h. et coll. 2011. « Burden of caregivers of patients with bipolar affective disorders », Am J Orthopsychiatry, 81, p. 139-148.

bleger, J. 1967. Symbiose et ambiguïté, Paris, Puf, 1981.

brun, A. ; roussillon, r. ; attigui, P. (sous la direction de) 2016. Évaluation clinique des psychothérapies psychanalytiques. Dispositifs individuels, groupaux et institutionnels, Paris, Dunod.

cook-darzens, S. 2007. «Les évaluations de l'approche multifamiliale », dans S. Cook-Darzens (sous la direction de), Thérapies multifamiliales. Des groupes comme agents thérapeutiques, Toulouse, érès, p. 57-78. 
federn, P. 1979. La psychologie du moi et les psychoses, Paris, Puf.

foulkes, S. H. 1975. Group Analytic Psychotherapy Methods and Principals, Londres, New-York, Gordon \& Breach.

fristad, m.a. ; gavazzi, s.m. ; mackinaW-koons, B. 2003. « Family psychoeducation: an adjunctive intervention for children with bipolar disorder », Biol Psychiatry, 53 (11), p. 1000-1008.

gex-fabry, m. ; guÉnoud, s. ; stauffer-Corminboeuf, m.J. et coll. 2015. « Group Psychoeducation for Relatives with Bipolar Disorder », J. of Nerv and Mental Disease 203, 9, p. 730-734.

henckes, N. 2015. «La psychiatrie de secteur, quelle histoire, quel avenir », Esprit, $\mathrm{n}^{\circ} 413$, p. $28-40$.

Jaitin, R. 1995. « Le porte-voix dans l'œuvre d'Enrique Pichon Rivière », Revue de psychothérapie psychanalytique de groupe, $\mathrm{n}^{\circ} 23$, p. 173-177.

Jaitin, R. 2002. "Théories et méthodes de formation à l'école de Pichon Rivière (Buenos Aires) ", Revue de psychothérapie psychanalytique de groupe, $\mathrm{n}^{\circ} 39$, p. 141-179.

Jarchov-Jadi, i. ; montag, g. et coll. 2013. Das Weddinger Modell, Berlin, Psychiatrie verlag $\mathrm{GmbH}$.

Joublin, H. 2005. Réinventer la solidarité de proximité. Manifeste de proximologie, Paris, Albin Michel.

1aronche, m. 2011. " on se sent si bien dans les phases euphoriques... », Le Monde du $11 / 06 / 2011$.

1e Cardinal, p. ; roelandt, J.-1. ; rafael, f. et coll. 2013. « Pratiques orientées vers le rétablissement et pair-aidance : historique, études et perspectives ", L'Info psychiat, 89, p. 365-370.

marchand, d. 2008. « À Propos de la parution de l'ouvrage Thérapies multifamiliales. Des groupes comme agents thérapeutiques, sous la direction de Solange Cook-Darzens, érès, coll. «Relations », 2007 : entretien avec Solange CookDarzens », Santé mentale au Québec, vol. 33, 1, p. 157-67.

masson, M. ; brun, M. 2015. "Psychiatrie et psychanalyse : le divorce est-il consommé ? À propos des “troubles bipolaires" ", L’Évol Psychiat, n 80 (2), p. 433-444.

m'bailar, k. ; gay, C. 2014. « Famille et troubles bipolaires », dans M.-L. Bourgeois, C. Gay et coll. (sous la direction de), Les troubles bipolaires, Paris, Lavoisier, p. 541-550.

machado-kupfer, m.-c. ; de 1aJonquière, 1. 2014. « Quelle place pour les parents d'enfants autistes dans le soin ? », Dialogue, $\mathrm{n}^{\circ} 206$, p. 11-22.

metz, c. ; nicot, c. ; bacqué, M.-F. 2017. " Support groups for parents with an adult child suffering from bipolar disorder », Psychodynamic Practice, $n^{\circ} 24(1)$, p. $40-55$.

mickelson, K.D. 1997. "Seeking social support: Parents in electronic support groups », dans S. Kiesler (sous la direction de), Culture of the Internet, Penn State University, p. 157-178.

nicot, C. 2015. Trouble bipolaire et qualité de vie : revue de la littérature, mémoire de Master recherche 2, sous la direction de M.-F. Bacqué, Université de Strasbourg. 
pavuluri, m.n. ; graczyk, p.a. ; henry, d.b. et coll. 2004. « Child-and familyfocused cognitive-behavioral therapy for pediatric bipolar disorder: development and preliminary results », J Amer Acad Child Adolesc Psychiatry, 43 (5), p. 528-537.

perlick, d.a. ; mik1Witz, d.J. ; lopez, n. et coll. 2010. « Family-focused treatment for caregivers of patients with bipolar disorder », Bipol Dis Sept, 12 (6), p. $627-37$.

Wallcraft, J. ; amering, m. ; freidin, J. et coll. 2011. « Partnerships for better mental health worldwide : WPA recommendations on best practices in working with service users and family carers », World Psychiat. 10, 3, p. 229-36.

West, a.e. ; Jacobs, r.h. ; Westerholm, r. et coll. 2009. " Child and familyfocused cognitive-behavioral therapy for pediatric bipolar disorder: pilot study of group treatment format », J Can Acad Child Adolesc Psychiatry 18 (3), p. 239-46.

ZendidJian, X. ; richieri, r. ; adida, M. et coll. 2012. " Quality of life among caregivers of individuals with affective disorders », J Affect Dis, 136, 3, p. 660- 665. 